\section{Regards sur l'économie allemande}

Bulletin économique du CIRAC

$93 \mid 2009$

Varia

\title{
La progression des bas salaires en RFA plaide pour un salaire minimum légal
}

\section{Gerhard Bosch}

Traducteur : Isabelle Bourgeois

\section{OpenEdition \\ Journals}

Édition électronique

URL : http://journals.openedition.org/rea/3900

DOI : $10.4000 /$ rea.3900

ISBN : 978-2-8218-0882-9

ISSN : 1965-0787

Éditeur

CIRAC

Édition imprimée

Date de publication : 1 octobre 2009

Pagination : 23-32

ISSN : 1156-8992

\section{Référence électronique}

Gerhard Bosch, « La progression des bas salaires en RFA plaide pour un salaire minimum légal »,

Regards sur l'économie allemande [En ligne], 93 | octobre 2009, mis en ligne le 01 octobre 2011,

consulté le 30 avril 2019. URL : http://journals.openedition.org/rea/3900; DOI : 10.4000/rea.3900 


\title{
La progression des bas salaires en RFA plaide pour un salaire minimum légal
}

\author{
Gerhard Bosch
}

En comparaison internationale, l'Allemagne a longtemps fait figure de pays où l'écart salarial est faible et où on ne compte que peu de bas salaires. II y a quelques années encore, on y menait d'intenses discussions pour savoir si oui ou non il fallait créer un segment à bas salaires pour soutenir l'emploi en général et plus particulièrement celui des actifs faiblement qualifiés. Et pourtant, un tel segment a toujours existé. Seulement, pendant longtemps, il n'a que peu varié, la part des actifs touchant une rémunération inférieure aux deux tiers du salaire médian s'établissant entre $13 \%$ et $14 \%$ du total. Or depuis le milieu des années 1990, le segment à bas salaires s'est étendu de manière significative sans qu'on s'en rende compte immédiatement, et sans apporter d'amélioration à la situation de l'emploi en général ni accroître les perspectives d'emploi des moins qualifiés. Par la suite, les réformes Hartz ont déclenché une nouvelle croissance de ce segment, politiquement voulue, cette fois, et cette tendance se poursuit.

Alors qu'en France et au Danemark, la proportion des actifs à bas salaires par rapport au nombre total des actifs occupés se situe depuis des années à un faible niveau en comparaison internationale, elle s'est considérablement accrue en Allemagne où elle dépasse même aujourd'hui le niveau enregistré au RoyaumeUni. Et l'écart avec les Etats-Unis s'est réduit lui aussi (Solow, 2008).

\begin{tabular}{|ccc|}
\hline \multicolumn{2}{|c|}{ Tableau 1 : Part des bas salaires en comparaison internationale (2005) } \\
USA : $25,0 \%$ & Royaume Uni : $21,7 \%$ & France : $11,1 \%$ \\
RFA : $22,0 \%$ & Pays-Bas : $17,6 \%$ & Danemark : $8,5 \%$ \\
\hline
\end{tabular}

Source : d'après Mason/Salverda, 2008.

Après avoir retracé l'évolution comme la structure du segment à bas salaires en Allemagne, nous en analyserons les causes puis résumerons les termes des débats actuels sur l'introduction d'un salaire minimum légal.

\section{Evolution et structure des emplois à bas salaires en RFA}

Depuis 1995, l'écart entre hauts et bas revenus n'a cessé de s'agrandir, comme le révèle la comparaison de la répartition des revenus corrigés de l'inflation en 1995, 2000 puis 2006 : alors que la part des revenus moyens se réduit, la part des revenus aux deux extrémités de l'échelle s'accroît. Et on voit se dessiner une nette hausse des emplois à bas salaires (salaires horaires bruts inférieurs à $6 €$ aux prix de 1995 et à $7 €$ aux prix de 2006). C'est cet écartement vers le haut et le bas qui est à l'origine de l'amenuisement du groupe des salaires moyens. Alors que celui-ci rassemblait encore plus de $63 \%$ de tous les actifs occupés en 1995, il s'est réduit de 11 points de pourcentage en 2006, les catégories des hauts et des bas revenus progressant d'autant (tableau 2).

Entre 1995 et 2006, les revenus réels moyens n'ont pratiquement pas progressé, du fait de la faible progression générale des salaires. Or si le gâteau ne s'agrandit guère et que ses parts sont réparties de plus en plus inégalement, il y a une forte probabilité pour qu'une partie des actifs occupés voie se réduire ses revenus, en valeur réelle, et peut-être même nominale. Or c'est exactement ce qu'on observe

Depuis 1995, la fourchette salariale s'est écartée vers le haut et le bas

Baisse des salaires jusque dans les catégories moyennes 
depuis 1995 en Allemagne. Dans le quartile inférieur, les revenus réels ont baissé de près de $14 \%$ depuis 1995 . Ils ont baissé également, bien que moins nettement, dans le deuxième quartile, alors qu'ils augmentaient dans les deux quartiles supérieurs (tableau 3). Entre 2000 et 2006, les revenus nominaux ont eux aussi baissé dans le quartile inférieur, c'est-à-dire que, en valeur absolue, les salaires étaient inférieurs au niveau de 2000. Le changement dans la distribution des revenus s'est donc fait largement en défaveur des catégories inférieures sur l'échelle des salaires. Mais si on considère les salaires réels, on observe qu'ils ont baissé jusque dans les catégories moyennes de revenus. En 2006, dans le quartile inférieur, le salaire horaire s'établit à $6,88 €$, soit en moyenne à 35 cents de moins qu'en 2000 . Alors que la perte nominale s'élève à 4,8 \%, la perte réelle atteint même 13,7 \%. En France, durant la même période, le SMIC horaire passait de $6,41 €$ à $8,27 €$, soit une hausse de près de $30 \%$. On comprend dès lors que soient apparus pour la première fois outre-Rhin des publications sous des titres comme : "La France, un modèle pour l'Allemagne » (Horn et al., 2008), alors qu'auparavant, c'était plutôt l'Allemagne qui avait valeur de modèle en France.

Tableau 2 : Répartition des salaires horaires (total des salariés ; ensemble de la RFA)

\begin{tabular}{|lccc|}
\hline Salaire horaire & $\mathbf{1 9 9 5}$ & $\mathbf{2 0 0 0}$ & $\mathbf{2 0 0 6}$ \\
Bas (<2/3 du médian) & $15,0 \%$ & $17,5 \%$ & $22,2 \%$ \\
Moyen (de $2 / 3$ à $4 / 3$ du médian) & $63,2 \%$ & $59,0 \%$ & $51,6 \%$ \\
Haut (> 4/3 du médian) & $21,8 \%$ & $23,6 \%$ & $26,3 \%$ \\
\hline
\end{tabular}

Source des données : SOEP (Sozio-ökonomisches Panel) 2006, calculs de l'IAQ. NB : Calcul différent des médians pour les Länder de l'ouest et de l'est.

Tableau 3 : Evolution 1995-2006 des salaires horaires nominaux et réels, par quartiles

\begin{tabular}{|l|ccc|cc|c|c|}
\hline & \multicolumn{3}{|c|}{ Salaire horaire nominal moyen } & \multicolumn{2}{c|}{$\begin{array}{c}\text { Evol. des salaires } \\
\text { horaires nominaux }\end{array}$} & $\begin{array}{c}\text { Salaires horaires réels } \\
\text { (aux prix de 1995) }\end{array}$ \\
& $\mathbf{1 9 9 5}$ & $\mathbf{2 0 0 0}$ & $\mathbf{2 0 0 6}$ & $\mathbf{1 9 9 5 - 2 0 0 0}$ & $\mathbf{2 0 0 0 - 2 0 0 6}$ & $\mathbf{2 0 0 6}$ & évol. 1995-2006 \\
$\mathbf{1}^{\mathrm{er}}$ quartile & $6,84 €$ & $7,23 €$ & $6,88 €$ & $5,8 \%$ & $-4,8 \%$ & $5,90 €$ & $-13,7 \%$ \\
$\mathbf{2}^{\text {ème }}$ quartile & $10,39 €$ & $11,15 €$ & $11,73 €$ & $7,3 \%$ & $5,2 \%$ & $10,06 €$ & $-3,2 \%$ \\
$\mathbf{3}^{\text {ème }}$ quartile & $13,11 €$ & $14,49 €$ & $16,02 €$ & $10,5 \% €$ & $10,6 \%$ & $13,74 €$ & $4,8 \%$ \\
$\mathbf{4}^{\text {ème }}$ quartile & $21,20 €$ & $22,79 €$ & $25,58 €$ & $7,5 \%$ & $12,3 \%$ & $21,94 €$ & $3,5 \%$ \\
Total & $12,88 €$ & $13,91 €$ & $15,05 €$ & $8,0 \%$ & $8,2 \%$ & $12,90 €$ \\
\hline
\end{tabular}

Source : SOEP 2006, calculs de l'IAQ.

Bas salaires : Le nombre des salariés touchant un bas salaire est passé outre-Rhin de 4,5 mil6,3 millions de salariés...

lions environ en 1995 à quelque 6,5 millions (2006), soit une hausse de $43 \%$. A relever que leur nombre a même augmenté de 700000 entre 2004 et 2006, c'està-dire durant une phase de croissance économique qui s'accompagne d'habitude d'une hausse des salaires y compris au bas de l'échelle. Tout porte à croire que cette augmentation est liée à l'adoption des lois Hartz à partir de 2003 (voir infra).

Comme il n'y a pas, en Allemagne, de salaire minimum uniforme et généralisé, l'écart de la fourchette des bas salaires est particulièrement prononcé. Ainsi, quelque 1,9 million d'actifs touchaient en 2006 un salaire horaire inférieur à $5 €$, soit nettement moins que les salariés payés au SMIC en France ou aux PaysBas, où le seuil plancher est supérieur à $8 €$ (Caroli/Gautié, 2008, Salverda, 2008).

... dont $54 \%$ occupent un emploi à temps partiel ou un mini-job
Alors qu'en 1995, les salariés occupant un emploi à temps plein étaient largement majoritaires ( $58 \%$ ) dans le segment des bas salaires, ils ne sont plus qu'un peu plus de $46 \%$ en 2006. Dans le même temps, la part des salariés occupant un emploi à temps partiel ou un mini-job a progressé : elle représente désormais $54 \%$ de ce segment, bien que, rapportée au total des actifs occupés, la part de ces salariés n'atteingne que $29,5 \%$ (tableau 4). Dans le segment des bas salaires, ce sont les mini-jobs qui enregistrent la plus forte progression, c'est-à-dire ces emplois rémunérés à moins de $400 €$ par mois. Ces salaires ne sont pas soumis à cotisations sociales. Seul l'employeur verse un forfait de $30 \%$, ces charges patronales correspondant grosso modo au montant des cotisations sociales versées en temps normal par le salarié. La part des mini-jobs dans le seg- 
ment des bas salaires a pratiquement doublé entre 1995 et 2006, ce qui reflète le rôle dévolu à ce type d'emplois dans le cadre de la politique pour l'emploi. Rapportée au total des actifs occupés, la part des mini-jobs a même presque triplé au cours de la même période, passant de $2,6 \%$ à $7,1 \%$.

Tableau 4 : Part respective des formes d'emploi dans le segment des bas salaires et l'emploi total en RFA (en \%)

\begin{tabular}{|lcccr|}
\hline & \% du segment des bas salaires & \multicolumn{2}{c|}{ \% dans l'emploi total } \\
& $\mathbf{1 9 9 5}$ & $\mathbf{2 0 0 6}$ & $\mathbf{1 9 9 5}$ & $\mathbf{2 0 0 6}$ \\
Temps plein & 57,9 & 46,2 & 79,0 & 70,6 \\
Temps partiel & 27,2 & 24,0 & 18,4 & 22,4 \\
Activité profession. « négligeable » (mini-jobs) & 14,9 & 29,7 & 2,6 & 7,1 \\
Total & 100,0 & 100,0 & 100,0 & 100,0 \\
\hline
\end{tabular}

Source : SOEP 2006, calculs de l'IAQ.

Le recul de la part des emplois à temps plein dans le segment des bas salaires ne signifie toutefois pas que ce type d'emploi serait moins concerné en 2006 qu'en 1995 par les bas salaires. Bien au contraire : alors qu'en 1995, un salarié en temps plein sur neuf touchait un bas salaire, cette proportion est passée en 2006 à un sur sept (tableau 5).

Temps plein : bas salaire pour un emploi sur sept

Tableau 5 : Emplois à bas salaires par catégorie d'emploi, RFA, 1995-2006 (en \%)

\begin{tabular}{|lcccc|}
\hline & $\begin{array}{r}\text { \% des bas salaires dans la catégorie } \\
\text { 1995 }\end{array}$ & \multicolumn{2}{c|}{$\begin{array}{c}\text { 1995-2006 : évol. de l'emploi (en valeur absolue) } \\
\text { Emplois à bas salaires }\end{array}$} & $\begin{array}{c}\text { Total emplois } \\
\text { Temps plein }\end{array}$ \\
Temps partiel & 11,0 & 14,3 & $+12,6 \%$ & $-13,5 \%$ \\
Mini-jobs & 22,2 & 23,4 & $+24,5 \%$ & $+18,0 \%$ \\
Total & 86,0 & 91,7 & $+181,2 \%$ & $+163,8 \%$ \\
\hline
\end{tabular}

Source : SOEP 2006, calculs de l'IAQ.

On considère généralement comme indispensable l'extension du segment des bas salaires pour ouvrir des possibilités d'intégration aux actifs peu qualifiés qui, sinon, resteraient exclus du marché de l'emploi. Et de fait, les calculs effectués

Dans l'emploi total, $45,6 \%$ des actifs peu qualifiés occupent un emploi à bas salaire par l'IAQ confirment que les actifs peu qualifiés entrent dans une proportion croissante dans la catégorie des bas salaires où ils sont surreprésentés : de 1995 à 2006, la part des emplois à bas salaires occupés par les actifs faiblement qualifiés s'est accrue de quelque $47 \%$. Or durant la même période, le taux de croissance enregistré par le groupe nettement plus important en nombre des actifs disposant d'une formation professionnelle a été bien supérieur, avec près de $70 \%$ (tableau 6).

Tableau 6: Part des emplois à bas salaires (en \%) par catégorie dans le total des actifs en RFA

\begin{tabular}{|llrrr|}
\hline \multirow{4}{*}{ Qualification } & $\mathbf{1 9 9 5}$ & $\mathbf{2 0 0 0}$ & $\mathbf{2 0 0 6}$ \\
& Sans formation professionnelle & $31,0 \%$ & $35,2 \%$ & $45,6 \%$ \\
& Formation prof. achevée & $13,6 \%$ & $17,8 \%$ & $23,1 \%$ \\
& Diplômés université/Fachhochschule & $6,3 \%$ & $6,1 \%$ & $6,1 \%$ \\
\hline \multirow{4}{*}{ Sexe } & Hommes & $7,4 \%$ & $9,9 \%$ & $14,2 \%$ \\
& Femmes & $25,0 \%$ & $26,6 \%$ & $30,5 \%$ \\
\hline \multirow{2}{*}{ Age } & $<25$ ans & $28,8 \%$ & $42,3 \%$ & $56,3 \%$ \\
& $25-34$ ans & $13,9 \%$ & $17,8 \%$ & $25,1 \%$ \\
& $35-44$ ans & $12,3 \%$ & $14,7 \%$ & $19,6 \%$ \\
& $45-54$ ans & $13,5 \%$ & $14,9 \%$ & $16,5 \%$ \\
\hline \multirow{2}{*}{ Nationalité } & $>55$ ans & $17,7 \%$ & $16,5 \%$ & $22,0 \%$ \\
\hline
\end{tabular}

Source : SOEP 2006, calculs de l'IAQ.

Quant à la structure par catégorie des salariés occupant un emploi à bas salaire (tableau 7), elle révèle par ailleurs que, en 2006, à peine un quart seulement de ces salariés appartiennent au groupe des actifs à faible qualification. A l'inverse,
Dans le segment des bas salaires, les $3 / 4$ des emplois sont occupés par des actifs qualifiés 
la part de ceux qui ont achevé leur formation professionnelle ou disposent d'un diplôme de l'enseignement supérieur a fortement progressé depuis 1995, passant de $66,5 \%$ à $73,6 \%$. Autrement dit, dans le segment des bas salaires, trois actifs sur quatre présentent une qualification professionnelle - un taux extrêmement élevé, même en comparaison internationale. Ainsi, aux Etats-Unis, environ 70 \% des actifs occupant un emploi faiblement rémunéré n'ont aucun diplôme ou tout au plus celui d'une high school, c'est-à-dire que leur niveau de qualification est inférieur à celui des sortants du système allemand de formation professionnelle (CBO, 2006).

Tableau 7 : Structure par catégorie des salariés occupant un emploi à bas salaire en RFA

\begin{tabular}{|c|c|c|c|c|}
\hline & & 1995 & 2000 & 2006 \\
\hline Qualification & $\begin{array}{l}\text { Sans formation prof. } \\
\text { Formation prof. achevée } \\
\text { Diplômés université/Fachhochschule }\end{array}$ & $\begin{array}{r}33,5 \% \\
58,6 \% \\
7,9 \%\end{array}$ & $\begin{array}{r}26,1 \% \\
66,4 \% \\
7,5 \%\end{array}$ & $\begin{array}{r}26,4 \% \\
67,5 \% \\
6,1 \%\end{array}$ \\
\hline Sexe & $\begin{array}{l}\text { Hommes } \\
\text { Femmes }\end{array}$ & $\begin{array}{l}27,5 \% \\
72,5 \%\end{array}$ & $\begin{array}{l}30,6 \% \\
69,4 \%\end{array}$ & $\begin{array}{l}31,9 \% \\
68,1 \%\end{array}$ \\
\hline Nationalité & $\begin{array}{l}\text { Allemande } \\
\text { Etrangère }\end{array}$ & $\begin{array}{r}88,5 \% \\
11,5 \%\end{array}$ & $\begin{array}{l}87,8 \% \\
12,2 \%\end{array}$ & $\begin{array}{l}87,9 \% \\
12,1 \%\end{array}$ \\
\hline
\end{tabular}

Source : SOEP 2006, calculs de l'IAQ. NB : Total des salariés, seuils salariaux différents à l'est et à l'ouest.

Cette forte disparité observée en Allemagne dans les profils des actifs occupant un emploi à bas salaire s'explique en partie par la persistance d'un taux de chômage élevé qui incite de plus en plus d'actifs qualifiés ou hautement qualifiés à accepter un emploi faiblement rémunéré. D'autre part, en Allemagne, le groupe des peu qualifiés est peu nombreux en comparaison internationale, du fait de la prépondérance du système de formation professionnelle.

\section{Les raisons de la hausse des inégalités sociales en RFA}

Fragilisation du système de partenariat social au fil de l'unification...

... et de l'ouverture à la concurrence des anciens services publics
Comment, dans un pays comme l'Allemagne, connu pour la puissance de ses syndicats et sa politique de réduction des inégalités sociales, ont pu se diffuser aussi rapidement faibles et très faibles salaires ? A mon avis, la raison principale réside dans la fragilité d'un modèle de partenariat conventionnel (« tarifaire ») exposé à la montée en puissance d'organisations concurrentes en marge du système établi. Comme il n'existe pas de planchers salariaux contraignants - qu'ils soient fixés via un salaire minimum légal ou une procédure d'extension -, rien n'interdit de verser des salaires inférieurs aux seuils des multiples conventions de branche. Jusqu'à l'Unité allemande, cela n'avait certes que des effets mineurs : $80 \%$ des entreprises étaient affiliées à une convention de branche, et les organisations syndicales étaient puissantes. Les entreprises non affiliées s'orientaient en fonction des conventions en vigueur dans leur branche pour ne pas voir migrer leurs salariés ou être en butte à des actions syndicales. Mais la montée du chômage après l'Unité a fortement réduit ces risques et, d'abord dans les nouveaux Länder, puis dans ceux de l'ouest aussi, un nombre croissant d'entreprises a quitté sa fédération patronale ou encore refusé d'y adhérer afin de pouvoir pratiquer des salaires inférieurs.

L'extension du segment des bas salaires observée dès le milieu de 1990 a fait tache d'huile jusque parmi les entreprises présentant un fort taux de syndicalisation. L'écartement croissant de la fourchette salariale se révéla ensuite de plus en plus incitatif pour délocaliser des tâches auparavant plus fortement rémunérées vers des branches ou des entreprises pratiquant des salaires inférieurs. L'inter- 
vention des pouvoirs publics a massivement soutenu cette évolution. Quant à la concurrence des secteurs non organisés [sur le modèle de la convention de branche ; IB], elle a été fortement avivée par une modification de la réglementation communautaire en matière de concurrence. La libre circulation des services permet de détacher en Allemagne des salariés rétribués selon les normes en vigueur dans leur pays d'origine. Sauf dans un secteur : le BTP où la loi allemande sur les travailleurs détachés (Arbeitnehmer-Entsendegesetz) a permis d'introduire des salaires minima légaux via la procédure d'extension appliquée aux conventions tarifaires conclues dans cette branche. Dans d'autres secteurs, comme la boucherie et les abattoirs, les salariés allemands, pourtant déjà peu rémunérés, ont été remplacés par des salariés sous contrat de louage de services (Werkvertrag) aux salaires encore plus bas (Czommer, 2007).

Plus profond encore est l'impact des directives de l'UE sur l'ouverture à la concurrence des anciens services publics (télécommunications, transports urbains, services postaux). Alors que dans la plupart des autres Etats membres, les nouveaux entrants sur ces marchés libéralisés sont tenus de respecter des lois ou conventions ayant force de loi et fixant des planchers salariaux, en Allemagne, ils sont libres de pratiquer tout salaire accepté par le marché. Cela invite les entreprises à développer des modèles économiques qui ne reposent plus sur l'innovation, mais sur la concurrence salariale, ainsi qu'on a pu l'observer dans le domaine des services postaux par exemple [voir sur ce dossier REA 85/08; IB].

A cette évolution s'est ajoutée l'impulsion politique donnée par les Lois Hartz, imposées par le gouvernement de coalition SPD/Verts en 2003 [voir REA 68/04; IB]. Parmi les modifications des dispositions légales, quatre surtout ont accru la pression sur les salaires. La première : les chômeurs de longue durée ne perçoivent plus qu'une indemnité forfaitaire minimale d'un montant récemment porté à $351 €$, à laquelle s'ajoutent diverses allocations complémentaires (dont une allocation loyer). Avant l'entrée en vigueur de cette disposition (2004), les chômeurs de longue durée percevaient un revenu de substitution calculé sur la base du revenu antérieur. Le changement de régime a surtout accru la pression sur les chômeurs qualifiés gagnant bien leur vie auparavant, les poussant à accepter un emploi mal rémunéré. La pression a été encore accrue par la seconde modification apportée : le renforcement des critères selon lequel un emploi est acceptable (zumutbar). Ainsi, les chômeurs doivent accepter l'emploi proposé, même si le salaire versé est inférieur (de $30 \%$ au maximum) aux usages en vigueur dans la région. Troisième modification : la levée des limitations de durée des missions d'intérim. Elle a donné aux entreprises la possibilité de remplacer, même définitivement, un certain nombre de leurs propres salariés par des travailleurs intérimaires moins rétribués. Contrairement à ce qui a été le cas en France, la disposition prévoyant l'égalité de traitement en matière de salaire n'a pas pu s'imposer en Allemagne, puisque les conventions tarifaires conclues dans l'intérim permettent aux agences de travail temporaire de déroger à ce principe d'égalité de traitement [sur le dossier intérim, voir REA 86/08; IB]. Entre-temps, les nombreuses conventions conclues dans le secteur prévoient, surtout pour les salariés nouvellement embauchés, des salaires plancher horaires nettement inférieurs au seuil des bas salaires; plus généralement, dans les secteurs qui ont classiquement recours au travail temporaire (comme la sous-traitance ou l'assemblage automobile, ou encore la construction mécanique), les salaires horaires des intérimaires sont nettement inférieurs à ceux des salariés de la branche (Weinkopf, 2006).

Quatrième modification : le relèvement de $300 €$ à $400 €$ par mois du salaire ouvrant droit au statut de mini-job. Certes, en principe, les 'mini-jobbers' jouissent eux aussi pleinement de la protection apportée par le droit du travail, celui-ci leur ouvrant droit au même salaire horaire que tout actif occupant un emploi similaire soumis à cotisations sociales. Mais dans la pratique, le statut dérogatoire instauré pour les mini-jobs prive souvent ceux qui les occupent des droits de tout salarié : les jours fériés, congés et congés maladie sont rarement rémunérés.
Extension du secteur des bas salaires, un choix politique aussi 
Temps partiel et mini-jobs dominent dans les services

Essor de l'intérim comme outil de flexibilité dans l’industrie

France, Pays-Bas, Danemark : des seuils plancher endiguent la concurrence salariale
C'est surtout dans le secteur des services, en particulier dans le commerce de détail ou la gastronomie, que de nombreux emplois à temps plein ou d'emplois à temps partiel soumis à cotisations sociales ont été divisés en plusieurs mini-jobs, moins coûteux pour l'employeur; les femmes sont alors les premières concernées. Dans ces secteurs, la moitié des salariés seulement $(47,4 \%)$ occupe aujourd'hui un emploi à temps plein; l'autre moitié se répartit à égalité entre un emploi à temps partiel ou un mini-job. De 1999 à 2007, la part du temps plein s'est réduite de 15 points de pourcentage, alors que celle des mini-jobs croissait de 20 points (Voss-Dahm, 2009).

Entre 2003 et 2008, le nombre des travailleurs intérimaires, en majorité des hommes, est passé d'environ 330000 à quelque 670000 (Bundesagentur für Arbeit, 2009). La hausse concerne surtout les branches à hauts salaires comme la métallurgie, où parfois des lignes de production entières ont été confiées à des intérimaires, aux salaires horaires inférieurs. Certes, au cours de la crise actuelle, de nombreux travailleurs temporaires ont été licenciés. Mais le secteur de l'intérim ne s'en montre pas moins très optimiste quant à l'avenir. Le président de la Bundesverband Zeitarbeit Personal-Dienstleistungen, l'une des fédérations de la branche, l'exprimait ainsi : " Aussi paradoxal que cela paraisse, la crise offre de vastes perspectives au secteur de l'intérim... Car de plus en plus d'entreprises clientes potentielles comprennent désormais l'importance de la flexibilité et donc de l'embauche de travailleurs temporaires - durant la crise, mais surtout après » (VBG, 2009).

Le mieux, pour comprendre la particularité des évolutions allemandes par rapport à celle d'autres pays, est de considérer l'architecture du système institutionnel de fixation des salaires. Le tableau 8 ci-dessous établit une comparaison de cette architecture dans 6 pays. Dans trois d'entre eux (France, Danemark, Pays-Bas), celle-ci est stable et se traduit par une couverture conventionnelle élevée. En France et aux Pays-Bas, son caractère contraignant résulte moins d'un fort taux de syndicalisation que du fort taux d'organisation des employeurs et, surtout, de l'utilisation fréquente de la procédure d'extension. A la différence des Pays-Bas, les syndicats sont toutefois très faiblement représentés dans quelques secteurs typiques du segment des bas salaires en France (comme le textile, l'habillement, le bâtiment ou, plus généralement, l'artisanat, le commerce et les services de proximité), de sorte que les salaires conventionnels y sont souvent inférieurs au SMIC ; ce dernier agit donc comme un salaire plancher. Dans les deux pays, on constate de facto deux types de salaires minima : le salaire minimum légal en tant que seuil plancher et, sur cette base, des conventions salariales 'étendues', très diverses selon les branches. Au Danemark, le caractère fortement contraignant des salaires est surtout la conséquence d'un taux de syndicalisation élevé. En outre, fédérations patronales et syndicats y ont décidé un salaire minimum conventionnel généralisé de $13,80 €$ dont les partenaires sociaux contrôlent efficacement le respect sans intervention de l'Etat (Westergaard, 2008). Dans ces trois pays, la concurrence salariale est ainsi endiguée par ces seuils plancher.

Tableau 8 : Taux de couverture conventionnelle, affiliation aux fédérations patronales, syndicalisation

\begin{tabular}{|c|c|c|c|c|c|c|c|c|c|c|}
\hline & $1-10$ & $11-20$ & $21-30$ & $31-40$ & $41-50$ & $51-60$ & $61-70$ & $71-80$ & $81-90$ & $91-100$ \\
\hline France & G & & & & & & & $\mathbf{U}$ & & $T(A)$ \\
\hline Pays-Bas & & & G & & & & & $\mathbf{U}$ & $\mathrm{T}(\mathrm{A})$ & \\
\hline Danemark & & & & & & $\mathbf{U}$ & & G & $\mathbf{T}$ & \\
\hline Allemagne & & & G & & & & $\mathbf{U}, \mathbf{T}$ & & & \\
\hline Royaume-Uni & & & G & $\mathbf{U}, \mathbf{T}$ & & & & & & \\
\hline Etats-Unis & $\mathbf{U}$ & $\mathbf{G}, \mathbf{T}$ & & & & & & & & \\
\hline
\end{tabular}

Source: Visser, 2008; Commission européenne, 2006.

Légende: $\mathbf{T}=$ Taux de couverture conventionnelle. $\mathbf{U}=$ Affiliation à des fédérations patronales rapportée au taux d'organisation des employeurs. G = Taux de syndicalisation (rapporté au taux d'organisation des salariés). $\mathbf{A}=$ La procédure d'extension s'applique à la plupart des conventions collectives. 
En Allemagne, le taux de couverture conventionnelle est tombé à $65 \%$ dans les Länder de l'ouest et même à $54 \%$ dans ceux de l'est, essentiellement parce que les entreprises ont quitté les fédérations patronales de branche ou que les entreprises nouvellement créées ont refusé de s'y affilier (Bosch/Weinkopf, 2007). C'est ainsi que se sont constituées, parallèlement au modèle tarifaire allemand qui prévaut dans ces branches où les syndicats sont puissants, de grandes zones blanches où le marché du travail n'est pas régulé et où se sont diffusés les bas salaires. Et pour réduire leurs coûts, de plus en plus d'entreprises affiliées à une fédération de branche délocalisent certaines activités au sein de petites et moyennes entreprises non affiliées. Or dans ces dernières, il n'y a généralement pas de conseil d'entreprise qui pourrait exercer, grâce à ses pouvoirs de codécision, le rôle d'instance de contrôle contre les pratiques de dumping salarial.

Dans plusieurs branches du secteur tertiaire présentant un faible taux d'organisation tant parmi les employeurs que parmi les salariés, ainsi par exemple le commerce de détail, les standards tarifaires étaient par le passé garantis surtout par la procédure d'extension appliquée aux conventions de branche. Or depuis plusieurs années, les fédérations patronales refusent de donner leur accord à l'extension des conventions qu'ils ont conclues, ce qui a réduit à néant l'un des piliers du système conventionnel allemand. Un dumping salarial presque sans limites dans ces secteurs a rendu hautement instables les institutions du système tarifaire allemand.

Les Etats-Unis et le Royaume-Uni présentent des zones blanches, c'est-à-dire sans conventions salariales, bien plus importantes encore qu'en Allemagne, étant donné que les conventions sont conclues presque exclusivement au niveau des employeurs. Les conventions généralisées, telles qu'elles s'appliquaient encore à l'ensemble d'une branche (Flächentarifverträge) au Royaume-Uni dans les années 1980, régulant les salaires, ou même aux Etats-Unis (pour les salaires des chauffeurs routiers, par exemple), n'ont pas survécu à la concurrence salariale. Néanmoins, l'instauration de salaires minima y a créé des seuils plancher ; mais ils se situent à un très bas niveau, surtout aux Etats-Unis.

\section{Le débat allemand sur les salaires minima légaux}

II y a quelques années encore, on n'évoquait l'instauration de salaires minima légaux que dans le secteur du BTP où était employée de manière croissante une main-d'œuvre issue des Etats du sud, du centre et de l'est de l'Europe, rémunérée selon les normes salariales en vigueur dans leur pays d'origine. Ces bas salaires constituaient non seulement une offre inférieure aux seuils fixés par les conventions de branche, mais ils généraient également, ce faisant, une distorsion de la concurrence entre les entreprises du secteur. En réaction, syndicats et fédérations patronales du BTP avaient alors adopté conjointement des salaires minima pour ses différents métiers et réussi en 1996, malgré la farouche opposition des confédérations patronales, à les rendre contraignants dans l'ensemble de la branche grâce à l'adoption de la Loi sur les travailleurs détachés (ArbeitnehmerEntsendegesetz). Depuis lors, ces salaires minima légaux de branche s'appliquaient à tous les salariés détachés sur un chantier en Allemagne par leur employeur sis dans un autre Etat membre de l'UE.

Ces dispositions particulières au BTP ont longtemps constitué une exception, les syndicats, les fédérations patronales et les partis politiques considérant que, hors cas particulier, les salaires doivent être fixés par les partenaires sociaux et que l'Etat n'est pas compétent en la matière. Ce consensus - fondé en droit sur l'autonomie que garantit la Constitution aux partenaires sociaux en matière de fixation des salaires et de conditions de travail -, a commencé à perdre de sa consistance quand il a fallu se rendre à l'évidence que le système du partenariat social n'était plus en mesure de fixer des planchers salariaux réellement contraignants pour les différents secteurs de l'économie. Les premiers à plaider
En RFA, le taux de couverture conventionnelle est tombé à $65 \%$

Dumping salarial sans limites dans le secteur tertiaire en RFA

Etats-Unis et Royaume-Uni ont instauré des seuils plancher

En 1996 ont été adoptés des salaires minima légaux dans le BTP

Une exception qui devrait devenir la règle générale? 
Extension des salaires minima légaux à d'autres branches : deux procédures 9 branches ont adopté
un salaire minimum,
d'autres sont candidates pour l'introduction d'un salaire minimum légal furent les syndicats des branches où se pratiquent les plus bas salaires, presque tous dans les services. Les puissants syndicats d'industrie s'y opposèrent dans un premier temps, redoutant qu'un salaire minimum légal ne déclenche une spirale tirant vers le bas le niveau élevé des salaires conventionnels de l'industrie; mais ils finirent par se rallier à cette revendication. Quant aux partis SPD et les Verts, ils ont opéré un revirement. Alors qu'avec les Lois Hartz de 2003, ils visaient l'extension du segment des bas salaires, en 2005 , à l'issue du mandat de la coalition gouvernementale SPD/Verts, ils sont entrés en campagne électorale en prônant l'adoption d'un salaire minimum légal uniforme et généralisé. Pour leur part, le patronat et les partis conservateurs (CDU/CSU et FDP) s'opposent toujours à l'institution de salaires minima légaux, considérant que ceux-ci détruisent de l'emploi. Actuellement, plus des deux tiers des Allemands soutiennent le projet de créer un salaire minimum légal, dont un nombre significatif d'électeurs des partis traditionnels.

Du fait des divergences existant entre les deux formations de la Grande coalition CDU/CSU et SPD (2005-2009), aucun accord n'a pu être trouvé sur l'adoption d'un salaire minimum légal universel sur le modèle britannique ou français. En lieu et place, il a été convenu de fixer des salaires minima légaux dans quelques branches. Le traité de coalition adopté à la fin 2005 prévoit deux procédures à cet effet [voir à ce sujet REA $88 / 08$; IB]. La première : syndicats et fédérations patronales peuvent créer des salaires minima dans le cadre d'une convention de branche conclue ad hoc. Si ces conventions sont conclues à l'échelle de l'ensemble de l'Allemagne et qu'elles couvrent $50 \%$ des salariés de la branche, le gouvernement fédéral peut leur conférer force de loi grâce à la procédure d'extension prévue dans le cadre de la Loi sur les travailleurs détachés. Toutefois, les conditions pour que cette procédure s'applique ne sont réunies que dans quelques secteurs ou branches. Dans d'autres, se caractérisant par une forte proportion d'emplois à bas salaires (la boucherie, par exemple), aucune convention ne se conclut à l'échelle de l'ensemble du pays, ce qui rend impossible l'adoption d'un salaire minimum légal. Dans d'autres encore, dont l'intérim, coexistent plusieurs conventions concurrentes comprenant des seuils plancher différents.

La deuxième procédure permettant d'instaurer des salaires minima légaux découle de la récente réforme (2009) de la Loi sur les conditions de travail minimales (Mindestarbeitsbedingungengesetz) de 1952. Dans les branches où les conditions décrites ci-dessus ne sont pas réunies, l'Etat peut fixer à l'avenir des standards minima. Cette procédure suit plusieurs étapes: dans un premier temps, une "Commission principale » (Hauptausschuss) composée de deux représentants respectivement des syndicats, des fédérations patronales, ainsi que des milieux scientifiques, et présidée par une personnalité indépendante, examine la question de savoir si on constate dans une branche donnée une situation "d'urgence sociale » (soziale Verwerfungen) requérant l'instauration de minima salariaux. Lorsqu'une telle situation est constatée, on procède à l'institution d'une Commission technique (Fachausschuss), composée de représentants du patronat et des syndicats des branches concernées, et chargée de se mettre d'accord sur un salaire minimum. Celui-ci est alors rendu contraignant (promulgation par décret). Pour faciliter la prise de décision, le président de chacune des deux commissions, une personnalité indépendante, dispose d'une voix prépondérante.

Au début de septembre 2009, la première procédure (extension d'une convention de branche) a permis d'instaurer des salaires minima dans 9 branches (1,849 million de salariés ; voir tableau 9). Dans la plupart d'entre elles, les minima est-allemands sont inférieurs à ceux de l'ouest. D'autres (formation continue, services de sécurité et de gardiennage, soins aux personnes) ont déposé un dossier pour bénéficier de cette procédure ; on ignore cependant le nombre de salariés qui y bénéficieraient d'un salaire minimum. La seconde procédure 
n'a pas jusqu'ici permis de fixer de salaire minimum, étant donné que la réforme de la Loi sur les conditions de travail minimales avait été successivement repoussée du fait de l'opposition du parti CDU/CSU. La Commission principale s'est constituée pour la première fois en septembre 2009, peu avant les élections au Bundestag du 27 septembre.

Tableau 9 : Salaires minima fixés par convention et étendus au moyen de la Loi sur les travailleurs détachés

\begin{tabular}{|c|c|c|}
\hline Branche et zone d'application & Catégorie de salariés concernée & SMIC horaire \\
\hline \multicolumn{3}{|c|}{ Gestion des déchets (130 000 salariés) } \\
\hline Ensemble du territoire & toutes & $8,02 €$ \\
\hline \multicolumn{3}{|l|}{ BTP (388 900 salariés) } \\
\hline $\begin{array}{l}\text { Länder de l'ouest + Berlin } \\
\text { Länder de l'est }\end{array}$ & $\begin{array}{l}\text { ouvriers } \\
\text { ouvriers professionnels } \\
\text { ouvriers et ouvriers professionnels }\end{array}$ & $\begin{array}{l}10,80 € \\
12,90 €(12,75 € \text { à Berlin }) \\
9,75 €\end{array}$ \\
\hline \multicolumn{3}{|c|}{ Services spécialisés dans le secteur minier (2 500 salariés) } \\
\hline & $\begin{array}{l}\text { non qualifiés } \\
\text { qualifiés }\end{array}$ & $\begin{array}{l}11,17 € \\
12,41 €\end{array}$ \\
\hline \multicolumn{3}{|c|}{ Services postaux (lettre) (140 000 salariés) } \\
\hline $\begin{array}{l}\text { Länder de l'ouest + Berlin } \\
\text { Länder de l'est }\end{array}$ & $\begin{array}{l}\text { distribution } \\
\text { autres } \\
\text { distribution } \\
\text { autres }\end{array}$ & $\begin{array}{l}9,80 € \\
8,40 € \\
9,00 € \\
8,00 €\end{array}$ \\
\hline \multicolumn{3}{|l|}{ Couverture (59 000 salariés) } \\
\hline Ensemble du territoire & toutes & $10,40 €$ \\
\hline \multicolumn{3}{|c|}{ Electricité (artisanat et industrie) (282 600 salariés) } \\
\hline $\begin{array}{l}\text { Länder de l'ouest } \\
\text { Länder de l'est + Berlin }\end{array}$ & $\begin{array}{l}\text { toutes } \\
\text { toutes }\end{array}$ & $\begin{array}{l}9,55 € \\
8,05 €\end{array}$ \\
\hline \multicolumn{3}{|c|}{ Entretien des bâtiments (700 000 salariés, dont 335300 sur des emplois soumis à cotisations sociales) } \\
\hline $\begin{array}{l}\text { Länder de l'ouest + Berlin } \\
\text { Länder de l'est }\end{array}$ & $\begin{array}{l}\text { toutes } \\
\text { toutes }\end{array}$ & $\begin{array}{l}8,15 € \\
6,58 €\end{array}$ \\
\hline \multicolumn{3}{|c|}{ Peinture/plâtrerie (111 400 salariés) } \\
\hline $\begin{array}{l}\text { Ensemble du territoire } \\
\text { Länder de l'ouest }\end{array}$ & $\begin{array}{l}\text { ouvriers non qualifiés } \\
\text { compagnons (artisans qualifiés) }\end{array}$ & $\begin{array}{l}9,50 € \\
11,25 €\end{array}$ \\
\hline \multicolumn{3}{|l|}{ Teinturerie (35 000 salariés) } \\
\hline $\begin{array}{l}\text { Länder de l'ouest } \\
\text { Länder de l'est }\end{array}$ & $\begin{array}{l}\text { toutes } \\
\text { toutes }\end{array}$ & $\begin{array}{l}7,51 € \\
6,36 €\end{array}$ \\
\hline Total salariés : 1849400 & & \\
\hline
\end{tabular}

Source : D’après www.mindestlohn.de.

La complexité de ce système de régulation offre de nombreuses possibilités de blocage aux milieux politiques, au patronat, ainsi qu'aux syndicats concurrents. Et les salaires minima ne sont adoptés qu'au compte-goutte. II est donc permis de craindre que va se constituer littéralement un patchwork se composant de salaires minima de niveaux disparates et comprenant de grandes zones blanches sans minima salariaux contraignants. L'Allemagne se différencie de la France ou du Royaume-Uni par l'absence d'un effet d'auto-renforcement par son application générale et simplifiée. On ne peut en effet présupposer que les salariés connaissent chacun dans son secteur ou activité le niveau du salaire minimum auquel ils peuvent prétendre ni qu'ils soient en mesure d'imposer leurs droits. Les instances de contrôle risquent d'être débordées par la pratique de la délocalisation des emplois mal rétribués vers des secteurs non régulés. Le projet de société largement partagé et qui consiste à barrer la route à des « salaires d'exploitation » court le risque de perdre toute légitimité puisqu'il ne parvient guère à endiguer la progression des bas salaires et que tout un chacun comprend que ce projet n'a pas le soutien du politique.
Mais pas de soutien politique pour barrer la route aux « salaires d'exploitation » 
POUR CONTRER CE RISQUE ET ENRAYER L'EXTENSION DU SEGMENT DES BAS SALAIRES, il convient de changer de politique. II faut instituer un salaire minimum légal uniforme et généralisé, un plancher unique s'appliquant à toutes les branches. II pourrait être fixé à $7,50 €$ dans un premier temps, puis atteindre progressivement le niveau pratiqué dans les autres pays occidentaux (comme la France ou les Pays-Bas). Sur cette base s'appuiera un second pilier, où les partenaires sociaux négocient les salaires conventionnels de branche; dans celles présentant une forte concentration de PME (traditionnellement peu organisées), ces salaires devraient être généralisés grâce à la procédure d'extension. Et, dans le cadre plus général d'un nouveau train de réformes sociales, il conviendrait de supprimer le statut d'exception des mini-jobs et de les réinsérer dans le régime commun des emplois soumis à cotisations sociales, car ce statut empêche souvent les employeurs de faire bénéficier ceux qui occupent un tel petit boulot des salaires ou prestations sociales prévus par la loi ou la convention de branche. II conviendrait en outre d'instaurer l'égalité de traitement (salaires) des travailleurs intérimaires afin de limiter la concurrence externe.

La transformation du système institutionnel allemand de fixation des salaires, fondé sur l'autonomie des partenaires sociaux, en un système mixte accordant une plus large place à l'Etat se heurte à une vive opposition et s'étendra de ce fait sur de longues années. A brève échéance, rien ne laisse donc entrevoir une baisse de la forte proportion des bas salaires en Allemagne.

Traduction: I. Bourgeois

\section{Indications bibliographiques:}

-Bosch G., Weinkopf C. (eds.), Arbeiten für wenig Geld : Niedriglohnbeschäftigung in Deutschland, Francfort/Main, 2007 (édition anglophone : Bosch G., WEINKOPF C. (eds.), Low-Wage Work in Germany, The Russell Sage Foundation Case Studies of Job Quality in Advanced Economies, New York, 2008

- BUndesagentur für ARBeIt, Statistik. Leiharbeitnehmer und Verleihbetriebe im 2. Halbjahr 2008, août 2009

- CARoli E., GautiÉ J. (eds.), Low-Wage Work in France, The Russell Sage Foundation Case Studies of Job Quality in Advanced Economies, New York, 2008

- CBO, Changes in the low-wage labor markets between 1979 and 2005, Congress of the United States, Congressional Budget Office, Washington, 2006

- Commission EuROPÉENNE, Industrial Relations 2006, Luxembourg

- Czommer L., « Wildwestzustände in Deutschland ? Einfacharbeitsplätze in der Ernährungsindustrie », in BOSCH G., WEINKOPF C. (eds.), op. cit.

- GrabKa M. M., FrICK J. R., « Schrumpfende Mittelschicht - Anzeichen einer dauerhaften Polarisierung der verfügbaren Einkommen ? ", in DIW-Wochenbericht n ${ }^{\circ} 10,2008$

- HoRn G., Joebges H., LogeAY C., STURN S., « Frankreich : Ein Vorbild für Deutschland ? Ein Vergleich wirtschaftspolitischer Strategien mit und ohne Mindestlohn », IMK Report n ${ }^{\circ} 31 / 2008$, Düsseldorf

- Salverda W., van Klaveren M., van der Meer M., Low Wage Work in the Netherlands, New York, Russell Sage Foundation, 2008

- Solow R. M., "Vorwort », in BosCh G., WeinKopf C. (eds.), op. cit.

-VeRWALTUNGSBERUfSGenOSSENSCHAFt (VBG), « Zeitarbeit in der Krise ? 2. Branchentreff Zeitarbeit der gesetzlichen Unfallversicherung VBG lockt etwas 180 Teilnehmer aus der gesamten Republik in den Landschaftspark Nord in Duisburg ». Téléchargé le 06-07-2009 (URL: www. presseportal.de/ pm/16868/1368845/verwaltungs_berufsgenossenschaft_vbg)

- VISSER J., Institutional Characteristics of Trade Unions, Wage Setting, State Intervention and Social Pacts (ICTWSS), an international database, Amsterdam Institute for Advanced Labour Studies (AIAS), Amsterdam, 2008

-Voss-DAHM D. «Warum Simone auch zukünftig weniger verdient als Simon: Ursachen geschlechtsspezifischer Ungleichheit im Einzelhandel », in LeHndoRfF S. (ed.), Abriss, Umbau, Renovierung? Studien zum Wandel des deutschen Kapitalismusmodells. Hamburg, 2009

-WEINKOPF C., Mindestbedingungen für die Zeitarbeitsbranche ? Expertise im Auftrag des Interessenverbandes Deutscher Zeitarbeitsunternehmen (iGZ), Institut Arbeit und Technik, Gelsenkirchen, 2006

-WestergaARd-Nielsen N. (ed), Low-Wage Work in Denmark, Russell Sage Foundation, NewYork, 2008. 